Check for updates

Cite this: RSC Adv., 2017, 7, 30963

Received 25th March 2017

Accepted 26th May 2017

DOI: $10.1039 / \mathrm{c} 7 \mathrm{ra0} 3482 \mathrm{~b}$

rsc.li/rsc-advances

\section{Spin-polarized charge trapping cell based on a topological insulator quantum dot}

\author{
Zhenhua Wu, $\dagger^{\star a}$ Liangzhong Lin, $\dagger^{\text {bd }}$ Wen Yang, ${ }^{c}$ D. Zhang, ${ }^{\text {b } C . ~ S h e n, ~}{ }^{b}$ W. Lou, ${ }^{b}$ H. Yin ${ }^{a}$ \\ and Kai Chang*b
}

We demonstrate theoretically that a topological insulator quantum dot can be formed via double topological insulator constrictions (TICS). The TICS are created by appropriate split-gate electrode patterns on the top of a $\mathrm{HgTe} / \mathrm{CdTe}$ quantum well (QW) with inverted band structures. In sharp contrast to conventional semiconductor quantum dots, the presence or absence of topological insulator edge states in the proposed quantum Hall bar system leads to distinct propagating behaviors. This topological insulator quantum dot can be used as a charge and/or spin carrier trap memory element with near perfect program/erase efficiency by properly adjusting the voltages applied to the split-gates. For completeness, we also demonstrate that a small perturbation of the Rashba spin orbit interaction (RSOI) or a magnetic field in the quantum dot does not destroy the topological edge states and has negligible impact on the on-(edge)-state transport behaviors of the quantum Hall bar.

\section{Introduction}

Manipulation of the electron and/or spin transport properties in topological insulators (TIs), ${ }^{1-3}$ such as $\mathrm{HgTe} / \mathrm{CdTe} \mathrm{QWs},{ }^{4-6}$ $\mathrm{Bi}_{1-x} \mathrm{Sb}_{x},{ }^{7} \mathrm{Bi}_{2} \mathrm{Se}_{3},{ }^{8} \mathrm{Bi}_{2} \mathrm{Te}_{3},{ }^{9}$ and $\mathrm{GaN} / \mathrm{InN} / \mathrm{GaN}$ and $\mathrm{GaAs} / \mathrm{Ge} /$ GaAs ultrathin quantum wells utilizing interface engineering, ${ }^{10,11}$ has attracted increasing interest over the past decade. ${ }^{12-21}$ In two dimensions (2D), a TI is also known as a quantum spin Hall (QSH) insulator, with insulating bulk states and gapless helical edge states. A QSH insulator state was predicted to occur in $\mathrm{HgTe}$ QWs with inverted band structures ${ }^{4,6}$ and was experimentally demonstrated. ${ }^{5}$ Since then, intensive efforts have been devoted to enriching the family of $2 \mathrm{D}$ QSH insulators by creating asymmetric InAs/GaSb quantum wells ${ }^{22,23}$ and $\mathrm{HgTe} / \mathrm{CdTe}$ or InSb/CdTe superlattices, ${ }^{24,25}$ by topological proximity effects in graphene, ${ }^{26}$ strain engineering, ${ }^{27-30}$ inducing defects, ${ }^{31}$ and applying external fields ${ }^{32}$ in certain twodimensional materials, by forming $2 \mathrm{D}$ III-Bi compounds, ${ }^{33}$ by forming $\alpha$-tin nanocrystals, ${ }^{34}$ and by chemical functionalization. ${ }^{35-38}$ So far QSH edge states have only been experimentally demonstrated in HgTe and InAs/GaSb quantum wells ${ }^{5,23}$ among those proposals. We hereby propose a HgTe based topological insulator quantum dot accounting for the practical feasibility. The gapless helical edge states in QSH insulators are spin

${ }^{a}$ MEDIT, Institute of Microelectronics, Chinese Academy of Sciences, Beijing 100029, P. R. China. E-mail: wuzhenhua@ime.ac.cn

${ }^{b}$ SKLSM, Institute of Semiconductors, Chinese Academy of Sciences, Beijing 100083, P. R. China.E-mail: kchang@semi.ac.cn

'Beijing Computational Science Research Center, Beijing 100094, P. R. China

${ }^{d} R \& D$ Center, Shenzhen Puyi Lighting Technology Ltd., Shenzhen 518000, P. R. China

$\dagger$ These authors contributed equally to this work. polarized and robust against non-magnetic impurities, crystalline defects, distortion of the surface, etc. due to the protection of time-reversal symmetry and a non-zero topological $Z_{2}$ invariant. ${ }^{1,39-42}$ These robust helical edge states provide new functional units for the construction of future nano-electronic devices.

The observation of conductance quantization of a nanoscaled constriction in two-dimensional electron gas (2DEG), the so-called quantum constriction, was a hallmark of mesoscopic transport physics. ${ }^{43,44}$ The constriction can be created by etching or depositing split-gate electrodes on top of the sample. Ever since then, constrictions in various systems have been studied extensively in both theoretical and experimental groups. Such constrictions are important building blocks of quantum dots and qubits for many potential device applications, e.g. single-electron transistors and quantum computers. ${ }^{45-49}$ After the recent discovery of the aforementioned new state of quantum matter, i.e. the TI state, the topological insulator constrictions (TICs) in the QSH system are of particular interest and offer an elegant way to control spin and charge transport. Currently, most works focus on the properties of a single TIC under different modulation mechanisms. ${ }^{50-56}$ It is highly desirable to study the impact of dual/multiple TIC configurations or more complex hybrid structures on the modulation, so as to achieve more flexible electric control of the carrier transport related to the topological helical edge states.

In this work, we investigate theoretically the carrier transport properties in experimentally realizable QSH systems. We propose a topological quantum dot formed in between two TICs in a $\mathrm{HgTe} / \mathrm{CdTe} \mathrm{QW}$ with an inverted band structure. The TICs are created using appropriate split-gate electrodes on top of the 
QW. The applied voltages determine the extension of the depletion regions underneath the split-gates, making it possible to effectively tune the width of each TIC independently. ${ }^{57}$ Rich transmission features will show up when changing the widths of the TICs or the Fermi energy. A minigap can be opened by the finite size effect at TICs, ${ }^{56}$ where the edge modes on opposite sides get coupled, resulting in a conductance dip. This feature enables us to switch on/off the edge channel by changing the TIC widths, i.e., by tuning the split-gate voltages. This configuration of a quantum dot sandwiched between two TICs can be used as a basic element in electronic storage devices. Utilizing the robust topological edge channels for switching on and the bulk gap for switching off gives rise to superior device reliability, endurance, and power consumption in the new proposed devices. Below we will illustrate these benefits in more detail. We also observed Fano-like or Fabry-Pérot-like resonances arising from interference in the quantum dot between the two TICs. The device performance is also robust against weak unintended magnetic fields and Rashba spin-orbit coupling from unintended electric fields.

The paper is organized as follows. In Section 2, we present the theory of electron tunneling through double TIC structures. In Section 3, we show the transmission, conductance, and charge distribution of the QSH bar system in order to illustrate the role of edge states in electronic storage devices. Finally, we give a brief conclusion in Section 4 .

\section{Theoretical model}

We consider a quantum dot with two TICs formed in a HgTe QSH bar, as shown schematically in Fig. 1(a). The desired inverted band structures of the HgTe QW in the low-energy regime are described by the so-called Bernevig-Hughes-Zhang (BHZ) model, ${ }^{4}$ i.e., a four-band Hamiltonian obtained from the eight-band Kane model by neglecting the light-hole and spinsplit bands. The size confinement of the QW is treated with a hard-wall potential $V, V(x, y)=V_{\text {ext }}$ for $y \in\left[Y_{\min }(x), Y_{\max }(x)\right]$ and $V(x, y)=\infty$ for $y<Y_{\min }(x)$ or $y>Y_{\max }(x)$, where $Y_{\min }(x)$ and $Y_{\max }(x)$ denote the lower and upper boundaries of the QSH bar. Allowing for the presence of a perpendicular magnetic field, Zeem energy splitting $g_{\mathrm{E}} \mu_{\mathrm{B}} \sigma \cdot \mathbf{B}$ and vector potential $\mathbf{A}$ are induced. The Landau gauge, $\mathbf{A}=\left(0, A_{y}, 0\right)$ is adopted in our calculation. Finally, by including the RSOI, such a single particle under the modulation of electric and magnetic fields can be described by a $4 \times 4$ Hamiltonian in the basis $|e \uparrow\rangle, \mid h h$ $\uparrow\rangle,|e \downarrow\rangle,|h h \downarrow\rangle$,

$$
\begin{aligned}
& H=H_{0}+V+H_{\mathrm{Z}} \\
& =\left(\begin{array}{cccc}
\varepsilon_{\mathrm{k}}+M(k) & A k_{-} & \mathrm{i} \alpha k_{-} & 0 \\
A k_{+} & \varepsilon_{\mathrm{k}}-M(k) & 0 & 0 \\
-\mathrm{i} \alpha k_{+} & 0 & \varepsilon_{\mathrm{k}}+M(k) & -a k_{+} \\
0 & 0 & -A k_{-} & \varepsilon_{\mathrm{k}}-M(k)
\end{array}\right) \\
& +\left(\begin{array}{cccc}
V+g_{\mathrm{E}} \mu_{\mathrm{B}} B_{z} & & 0 & 0 \\
& V+g_{\mathrm{HH}} \mu_{\mathrm{B}} B_{\mathrm{z}} & V-g_{\mathrm{E}} \mu_{\mathrm{B}} B_{z} & \\
& 0 & & V-g_{\mathrm{HH}} \mu_{\mathrm{B}} B_{z}
\end{array}\right),
\end{aligned}
$$

(a)

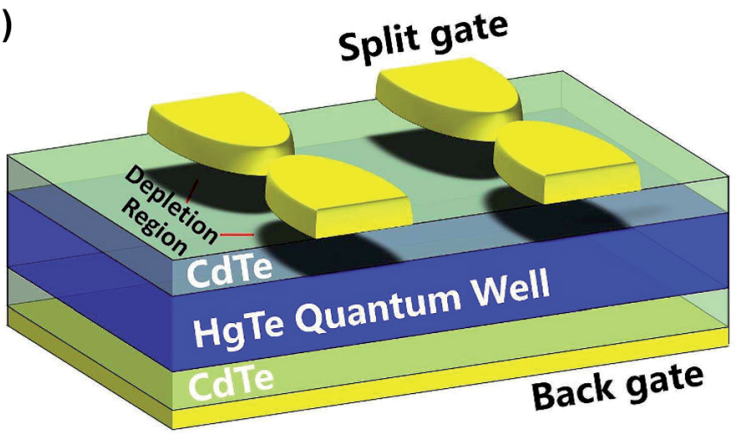

(b)

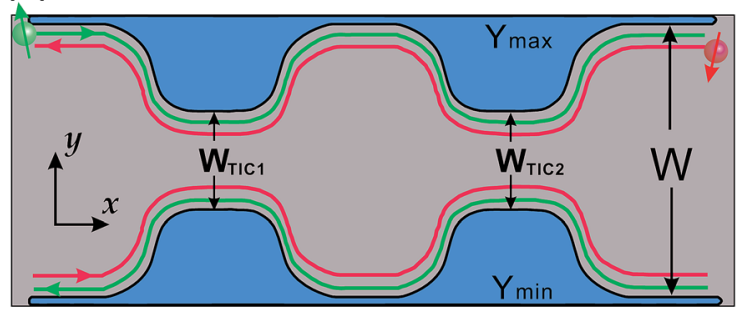

(c)

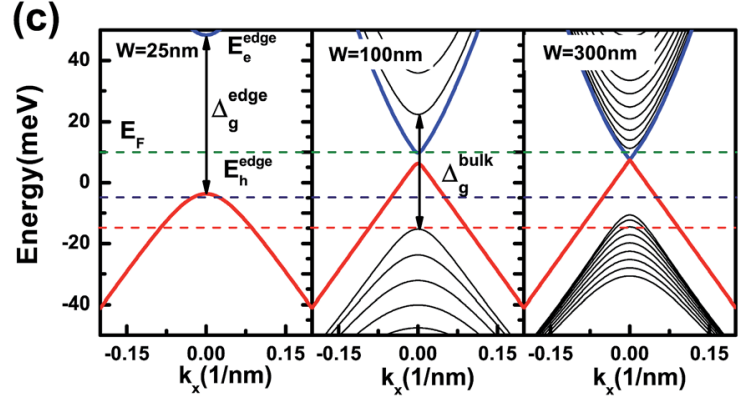

Fig. 1 (a) Schematic diagram of the HgTe quantum well based Hall bar structure with dual TICs. The HgTe quantum well is grown in the [001] direction with a channel along the [100] crystallographic direction. (b) Schematic illustration of spin-polarized QSH edge states. $W_{\mathrm{TIC1}}\left(W_{\mathrm{TIC} 2}\right)$ is the width of the first (second) TIC. The green/red lines indicate the trajectories of spin-up/spin-down carriers via the QSH edge states. (c) The energy dispersions of the HgTe QSH system with different QW widths. The thickness of the HgTe well is $7 \mathrm{~nm}$. The dashed lines indicate the positions of the Fermi energies chosen for the calculations in Fig. 2.

where $k=\left(k_{x}, \pi_{y}\right)$ is the in-plane momentum of electrons, $\pi_{y}=k_{y}+\frac{e}{h} A_{y}, \varepsilon_{\mathrm{k}}=C-D\left(k_{x}{ }^{2}+\pi_{y}{ }^{2}\right), M(k)=M-B\left(k_{x}{ }^{2}+\pi_{y}{ }^{2}\right), k_{ \pm}=$ $k_{x} \pm \mathrm{i} \pi_{y}, \alpha$ is the RSOI strength, and $A, B, C, D$, and $M$ are the parameters describing the band structure of the HgTe/CdTe QW. Note that the QSH state and band insulator state are characterized by the sign of the parameter $M$, which is mainly determined by the thickness of the $\mathrm{HgTe} / \mathrm{CdTe}^{\mathrm{Q}} \mathrm{W}^{4,5}$ as well as a perpendicular electric field. ${ }^{6}$ For negative (positive) $M$, the QW is in the QSH (bulk insulator) state. For a quasi-onedimensional (Q1D) QSH bar system, shown in Fig. 1(b), the transport property can be obtained by discretizing the Q1D system into a series of transverse strips along the transport direction with sharply narrowed widths at the TICs controlled by the split-gate modulation. Using scattering matrix theory, ${ }^{\mathbf{1 6}}$ the transmission amplitudes from the $m$-th input mode to the $n$ th output mode $t_{n \mathrm{R} ; m \mathrm{~L}}$ are obtained. We can obtain the total 
conductance of the QSH system at a temperature of zero from the Landauer-Büttiker formula,

$$
G^{0}\left(E_{\mathrm{F}}\right)=G_{0} \sum_{m \in \mathrm{L}, n \in \mathrm{R}} \frac{\nu_{n}^{\mathrm{R}}}{\nu_{m}^{\mathrm{L}}}\left|t_{n \mathrm{R} ; m \mathrm{~L}}\left(E_{\mathrm{F}}\right)\right|^{2},
$$

where the summation is over all propagation modes in the input/output leads that connect to the left/right of the quantum dot, and $G_{0}=e^{2} / h$ is the conductance unit. At a finite temperature $T$, the ballistic conductance can be written as

$$
G\left(E_{\mathrm{F}}\right)=\int\left(-\frac{\partial f(E)}{\partial E}\right) G^{0}\left(E_{\mathrm{F}}\right) \mathrm{d} E,
$$

where $f(E)=\left\{1+\exp \left[\left(E-E_{\mathrm{F}}\right) / k_{\mathrm{B}} T\right]\right\}^{-1}$ is the Fermi-Dirac distribution.

\section{Results and discussion}

In our proposal, the HgTe quantum well is grown in the [001] direction with a channel along the [100] crystallographic direction. The thickness of this HgTe well is $7 \mathrm{~nm}$, guaranteeing the existence of band inversion. ${ }^{4-6}$ The CdTe barrier is wider than $20 \mathrm{~nm}$, at which value the tunneling through CdTe layers ${ }^{24}$ can be well suppressed. Finally the QSH states can be realized for both electron and hole branches. We start by investigating the carrier transport properties in this QSH bar system without

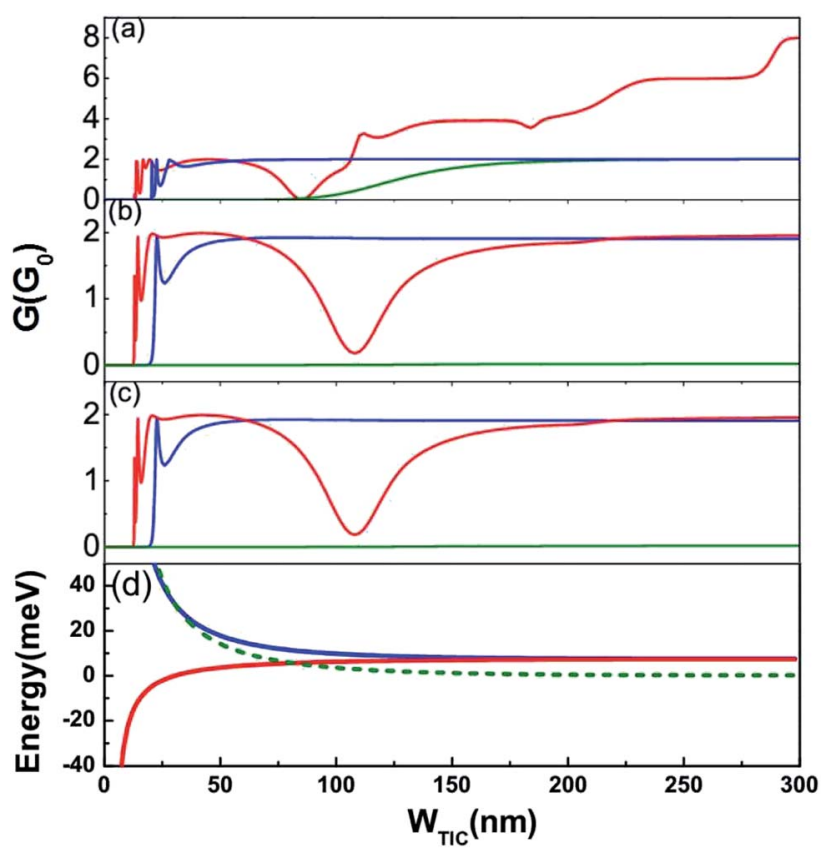

Fig. 2 (a)-(c) The conductance as a function of the width of the TICs for three different Fermi energies $E_{\mathrm{F}}=10 \mathrm{meV}$ (green lines), $-5 \mathrm{meV}$ (blue lines), and $-15 \mathrm{meV}$ (red lines). (a) The widths of dual TICs are changed simultaneously $\left(W_{\mathrm{TIC} 1}=W_{\mathrm{TIC} 2}=W_{\mathrm{TIC}}\right)$. (b) The width of the second TIC is fixed $\left(W_{\text {TIC2 }}=50 \mathrm{~nm}\right)$ and the width of the first TIC is changed $\left(W_{\mathrm{TIC} 1}=W_{\mathrm{TIC}}\right)$. (c) The width of the first TIC is fixed $\left(W_{\mathrm{TIC1}}=\right.$ $50 \mathrm{~nm})$ and the width of the second TIC is changed $\left(W_{\mathrm{TIC} 2}=W_{\mathrm{TIC}}\right)$. The width of the QSH bar is always $W=300 \mathrm{~nm}$. (d) The conduction band (blue line), valence band (red line), and energy gap (green dashed line) in the edge-state spectrum as a function of the width of the TICs.
RSOI or magnetic fields. Fig. 2(a)-(c) show the conductance variations with the dual TIC width modulations in three different ways, i.e. varying the widths of dual TICs simultaneously (a), and fixing the width of one TIC and varying the width of the other one (b and c). Due to the finite size effect, ${ }^{55,56}$ a finite gap can be opened in the energy spectra (see Fig. 1(c)) and the gap keeps increasing when reducing the width of the TICs as shown in Fig. 2(d). The electrostatic control of the position of the chemical potential with respect to the Dirac point allows for precise modulation of the transport properties. If the Fermi energy locates in this opened energy gap, there is no available propagating mode in the TIC region. Instead, carriers move in evanescent modes that decay rapidly in the TIC region, leading to the suppression of transmission. For extremely narrow TICs, we can always observe vanished conductance, as shown in Fig. 2(a), regardless of the incident Fermi energy we choose, since the gap is very large. For the green curve, i.e. $E_{\mathrm{F}}=$ $10 \mathrm{meV}$, the Fermi energy is close to the Dirac point of the QSH TI state. Even though only a small gap is opened when the widths of the TICs are controlled to just below $100 \mathrm{~nm}$, the Fermi energy has already located in this gap, consistent with the conductance drop observed in our calculations. For the blue curve, i.e. $E_{\mathrm{F}}=-5 \mathrm{meV}$, the Fermi energy lies far from the Dirac point of the QSH TI state. Therefore, we need a much larger gap in the TIC region with a further reduced width to block the edge channel. Note that the Fermi energy of the blue curve still does not touch the edge of the bulk subbands (bulk propagating modes) even when the TICs are as wide as the leads. In the above two cases, only the edge state contributes to the transport and thus the conductance saturates to the value of $2 G_{0}$ as we relax the TICs. Note that when the incident energy is fixed to $E_{\mathrm{F}}=-15 \mathrm{meV}$ the Fermi level is so far from the Dirac point that it is able to cross several bulk propagating subbands as the widths of the TICs get larger (see Fig. 1(c)). As we expect, the conductance does not saturate with the width of the TICs but exhibits a step-like feature, which corresponds to the opening of new bulk propagating modes in the TIC regions as they become wider. Furthermore we can observe a dip in the conductance curve at around $W \approx 85 \mathrm{~nm}$. The conducting channels come from both the QSH TI edge states and the bulk propagating states; the wave functions of the QSH TI states are squeezed in the central quantum dot region and couple with the bulk states in the narrow TICs. Due to the destructive quantum interference when the charge carriers transmit or reflect at TICs, carriers are localized in the quantum dot without transmission. In Fig. 2(b) and (c) we confirm that each single TIC can effectively block the carrier transport, making our proposed TI quantum dot able to work as an electronic storage device, which we will discuss in more detail later.

To confirm the analytical prediction of carrier localization, we plot the density distribution of both topological edge states and bulk states for the three cases in Fig. 3(a)-(c), corresponding to the dips in Fig. 2(a)-(c), respectively. The Fermi energy is $E_{\mathrm{F}}=-15 \mathrm{meV}$, and the widths of dual TICs are as follows, (a) $W_{\text {TIC1 }}=W_{\text {TIC2 }}=85 \mathrm{~nm}$, (b) $W_{\text {TIC1 }}=108 \mathrm{~nm}, W_{\text {TIC2 }}=50 \mathrm{~nm}$, and (c) $W_{\mathrm{TIC} 1}=50 \mathrm{~nm}, W_{\mathrm{TIC} 2}=108 \mathrm{~nm}$. Our calculations demonstrate the localized bound states in the quantum dot. Carriers 

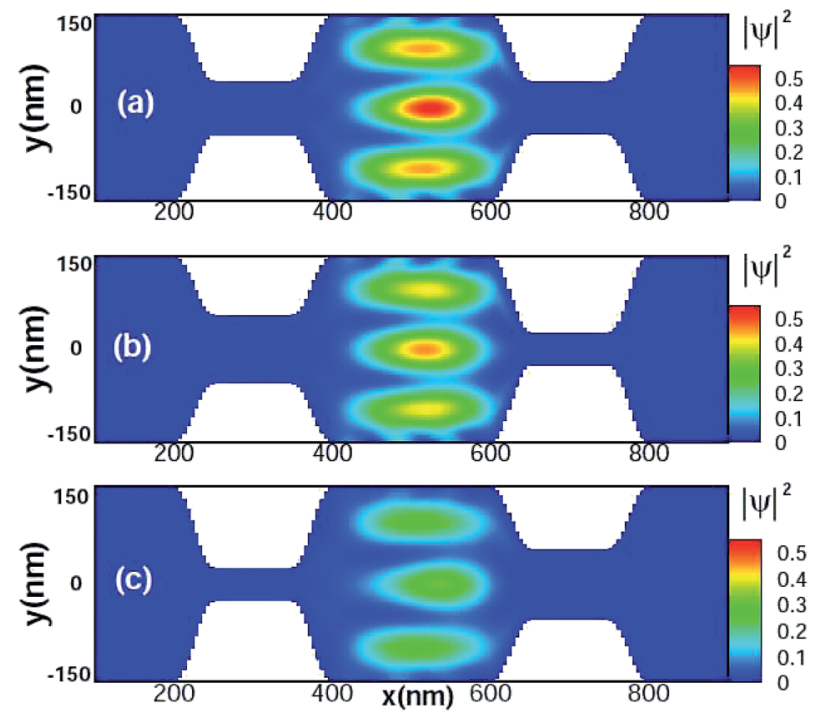

Fig. 3 The density distribution of the carrier in both the edge states and bulk states. The Fermi energy is fixed at $E_{\mathrm{F}}=-15 \mathrm{meV}$. The widths of the TICs are as follows: (a) $W_{\mathrm{TIC1}}=W_{\mathrm{TIC} 2}=85 \mathrm{~nm}$; (b) $W_{\mathrm{TIC} 1}=$ $108 \mathrm{~nm}, W_{\mathrm{TIC} 2}=50 \mathrm{~nm}$; (c) $W_{\mathrm{TIC} 1}=50 \mathrm{~nm}, W_{\mathrm{TIC} 2}=108 \mathrm{~nm}$.

can be confined in these coupled quantum states with appropriate TIC widths and Fermi energies. Despite the similarity of the charge trapping phenomenon to conventional quantum dots, the physical content is different in our system. In our TI quantum dot, the bound states arise from the coupling between one topological edge state and the bulk states and thus the conductance shows a single dip in Fig. 2(a)-(c). In the case of common quantum confinement of double barriers, the conductance exhibits oscillating behaviors with multiple local minima ${ }^{58}$ arising from the formation of Fabry-Pérot modes. We can adiabatically change the Fermi energy to approach such conditions of charge trapping. This provides the possibility of storing information in these coupled quantum states. However, there are some difficulties in realizing this application, which are the following. (1) It is difficult to break the edge channel via coupling and (2) the transition from the trapping phase to the transferring phase is rather smooth. (3) There is additional scattering between the bulk states outside of the depletion regions and the unexpected defect/trap states in the depletion regions, which may decrease the trapping efficiency of the carriers and jeopardize the validity of the hard-wall confinement potential assumption, and (4) the new mechanism of bound states in the quantum dot does not give rise to some functional benefits. For the purpose of making an electronic storage element, a very sharp transition is required to boost the device performance, e.g. a quick switch, large on/off ratio, low standby power, etc.

To find a possible solution, we consider the carrier transport purely in the helical edge channels by tuning the Fermi energy to $E_{\mathrm{F}}=-5 \mathrm{meV}$. The bulk subbands are too far from this Fermi energy to contribute to carrier transport. In the absence of RSOI or a magnetic field, we obtain a block diagonal Hamiltonian leading to the time-reversal symmetry for the upper and lower 2 $\times 2$ blocks. Therefore we can calculate the carrier transport of the spin-up or down state separately. Fig. 4 shows the density distribution of the helical edge states in the system for different TIC configurations. For a clear physical picture, we only take the spin-up carriers into consideration. In Fig. 4(a), the TICs are adequately wide with remaining edge channels in the whole QSH bar device region including the central quantum dot and dual TICs. The carriers propagate along the edge channel and perfectly transport through the topological quantum dot. If the second TIC is blocked, as in Fig. 4(b), one can see clearly that the carriers from the left incident lead can transport along the top edge into the quantum dot and be reflected to the opposite edge with the same spin orientation due to the helicity of the topological edge states. Then carriers leak out of the quantum dot easily, also via the edge channels. Since the width of the first TIC is wide enough, the coupling between the top and bottom helical edge states is very weak. Carriers can transport freely in these edge channels that are well kept in the TICs. If we reduce the width of the first TIC as we did for the second TIC, the coupling between the edge states in the opposite edges becomes spectacular, as we can see in Fig. 4(c). The maximum local probability $\left|\psi_{\text {TES }}{ }^{\sigma}\right|^{2}(\sigma=\uparrow, \downarrow)$ is approximately 0.17 between the dual TICs which is significantly enlarged with respect to the configuration shown in Fig. 4(b), in which the maximum local probability $\left|\psi_{\text {TES }}{ }^{\sigma}\right|^{2}(\sigma=\uparrow, \downarrow)$ is about 0.09 . This gap indicates that the carriers cannot reflect back to the left incident lead freely and tend to be stored in the quantum dot. If we keep reducing the width of the TIC, the coupling is strong enough to break the topological edge modes and the first TIC is blocked as well as the second TIC. One can first apply the pinch-off voltage on the split-gate to block the second TIC, after the charges are
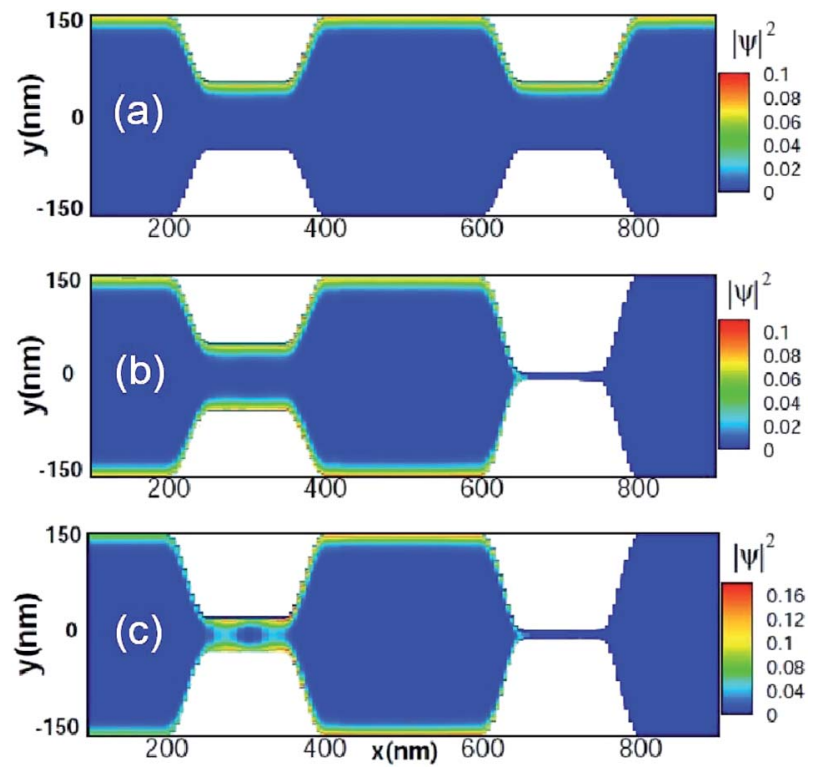

Fig. 4 The density distribution of the carrier in the edge states. The Fermi energy is fixed at $E_{\mathrm{F}}=-5 \mathrm{meV}$. The widths of TICs are as follows: (a) $W_{\mathrm{TIC} 1}=W_{\mathrm{TIC} 2}=100 \mathrm{~nm}$; (b) $W_{\mathrm{TIC} 1}=100 \mathrm{~nm}, W_{\mathrm{TIC} 2}=10 \mathrm{~nm}$; (c) $W_{\mathrm{TIC} 1}=50 \mathrm{~nm}, W_{\mathrm{TIC} 2}=10 \mathrm{~nm}$. 
injected from left lead via the spin-polarized helical edge channel, and then block the first TIC with the same technique.

In sharp contrast to conventional semiconductor quantum dots, the quantum states in our topological quantum dot are fully spin-polarized helical edge states at an appropriate Fermi energy in the bulk gap (in our discussion, $E_{\mathrm{F}}=-5 \mathrm{meV}$ ). The trapped charges show ring-like density distributions near the boundary as we demonstrated in Fig. 4(c). More importantly, the spin-angular momentum locking, determined by the $\mathrm{BHZ}$ Hamiltonian, gives rise to spin-polarized charge currents, i.e. the trapped spin-up carriers rotate clockwise and spin-down carriers rotate counterclockwise without spin flip or spin relaxation. ${ }^{59}$ If we inject spin polarized currents into the quantum dot, the polarized spin can be stored as well as the charge in the form of persistent spin/charge currents accounting for the topological protected edge states, which are robust against local perturbations. Note that Klinovaja, et al., demonstrated a novel all-electric-controlled spin filter in the same frame of TIC in two dimensional TIs. ${ }^{56}$ This can be used to generate the desired spin-polarized current. More importantly, this is also compatible with the topological quantum dot charge storage elements proposed in this work and people can dramatically reduce the integration difficulties in making a complete setup. This feature sheds new light on constructing charge/spin trapping memory elements for both electronic and spintronic devices.

To proceed further, we examine the impact of the aforementioned important HgTe QW band parameter $M$. As we have discussed, $M$ determines the presence of TI edge state or only trivial bulk insulator (BI) state. ${ }^{4}$ Modulation of the parameter $M$ is achieved mainly by changing the HgTe QW thickness and/or by tuning the bottom gate voltage. The bottom gate is commonly used technology in semiconductor nano-devices, giving us a practical way to switch the system between the TI and BI states. In Fig. 5(a)-(c) we show the QD conductance $G$ as a function of the Fermi energy $E_{\mathrm{F}}$ with different values of the band parameter $M$. The different tunneling process between BI $(M>0)$ and TI $(M<0)$ can be observed. In all three cases, the widths of the two TICs are fixed to $100 \mathrm{~nm}$. For the BI system, the conductance vanishes when the incident Fermi energy was located in the gap of the TICs due to the absence of propagating modes. For higher Fermi energies, conductance plateaus appear as well as many oscillations. Each plateau corresponds to the opening of a new bulk subband in the TICs. The oscillations originate from the Fabry-Pérot interference between the transmitted and reflected carriers in the quantum dot. For TI systems, the resonances are observed even when the Fermi energies are located not only in the bulk-subband energy regions but also in the small TI edge gap. When the incident Fermi energies are located in the bulk gap but not in the edge gap of the TICs, the transmission is nearly perfect, accounting for the helical edge channels. The oscillation of the transmission is smeared out since the backscattering process is suppressed, i.e. few Fabry-Pérot modes are formed. The switching between the TI state and BI state leads to a strongly enhanced on/off ratio of this QSH bar device. This observation

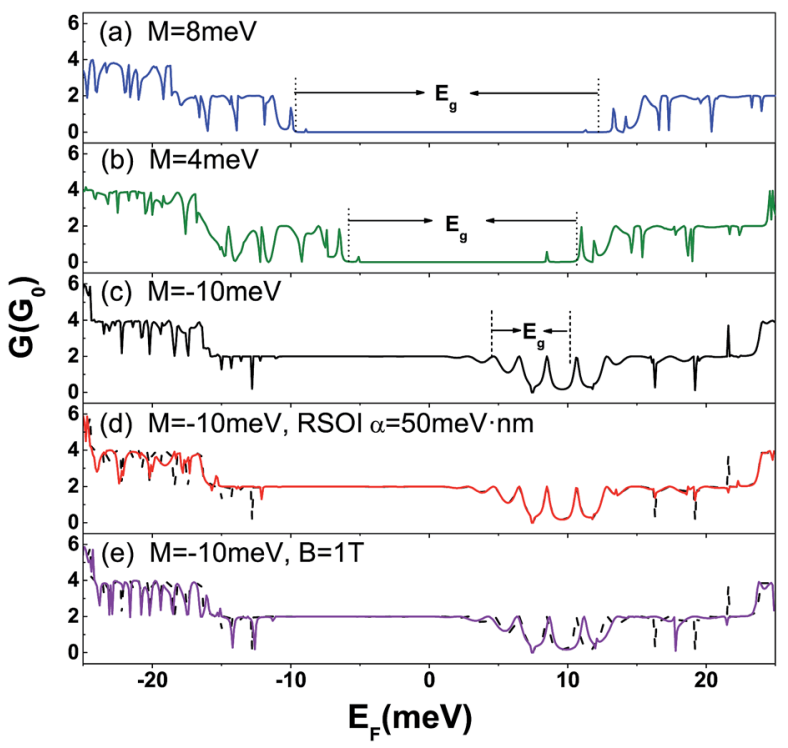

Fig. 5 Fermi energy dependence of the QD conductance with fixed TIC widths $\left(W_{\mathrm{TIC1}}=W_{\mathrm{TIC} 2}=100 \mathrm{~nm}\right)$. Panels (a) $-(\mathrm{c})$ show the switch between $\mathrm{BI}$ and $\mathrm{TI}$ determined by different values of the band parameter $M$ without considering RSOI or magnetic field perturbations. (a) $M=8 \mathrm{meV}$, (b) $M=4 \mathrm{meV}$, and (c) $M=-10 \mathrm{meV}$. Panels (d) and (e) are obtained based on almost the same settings as (c) except for including (d) RSOI $\alpha=50 \mathrm{meV} \mathrm{nm}$ (red line) or (e) a magnetic field $B=1 \mathrm{~T}$ (purple line). The black dashed lines indicate $\alpha=0$ and $B=0$ in the QD.

provides us with an additional way of controlling the carrier transport properties in the proposed HgTe QSH bar device.

So far, we have studied the carrier transport through the proposed QSH bar system exclusively under electric modulations. For completeness, we further investigate the carrier transport through the HgTe QSH bar in the presence of RSOI or a magnetic field in the quantum dot. Fig. 5(d) shows the conductance as a function of the incident Fermi energy in the presence/absence of RSOI. Since the TI edge states are immune to perturbations of RSOI, the conductance curves at different RSOI strengths ( $\alpha=0$ and $\alpha=50 \mathrm{meV}$ ) are almost the same in the energy region $-10 \mathrm{meV}<E_{\mathrm{F}}<15 \mathrm{meV}$, where the conducting channels only come from the TI edge states. If the Fermi energy locates out of the bulk band gap, i.e., $E_{\mathrm{F}}<-10 \mathrm{meV}$ or $E_{\mathrm{F}}>15$ $\mathrm{meV}$, the bulk modes are opened in the quantum dot. The RSOI would induce a spin splitting of the bulk subbands, resulting in different Fermi wave vectors $k_{\sigma}(\sigma=\uparrow, \downarrow)$ for spin-up and spindown carriers. However the spin splitting is quite small, so one can expect that this spin splitting only leads to slightly different behaviors compared to the case without RSOI in the quantum dot, and this is clearly reflected in Fig. 5(d).

Finally, we consider an external magnetic field applied perpendicularly to the HgTe quantum dot. Fig. 5(e) shows the conductance as a function of the incident Fermi energy $E_{\mathrm{F}}$ for $B=1 \mathrm{~T}$ and $B=0 \mathrm{~T}$. Interestingly, we find that the positions of the resonance conductance peaks are obviously shifted in a finite magnetic field of $B=1 \mathrm{~T}$. This is because the magnetic field breaks the time-reversal symmetry and shifts the Dirac 
point in the energy spectrum. ${ }^{59}$ Beyond the energy range with only a TI edge state, the conductance behaviors are completely different when we apply a finite magnetic field $(B=1 \mathrm{~T})$. More oscillations and sharp dips are observed in our calculations. These dips indicate strong backscattering in the quantum dot. This can be understood by the semi-classical picture; the magnetic field can bend the trajectory of the carriers in favor of cyclotron motion. Charge carriers have more chance to meet with each other before transmission through the quantum dot, and this will enhance the interference between different modes in the quantum dot. If the magnetic field is very large, the cyclotron orbit radius is smaller than the size of the quantum dot and the conductance can be fully suppressed. For small RSOI and magnetic fields, as we employed in above simulation, the TI edge states are well preserved. All of the aforementioned features needed to make a spin-polarized charge trapping device element are retained in the proposed quantum dot even in the presence of a small RSOI and/or a magnetic field.

\section{Conclusions}

In summary, we have theoretically investigated the carrier transport through a topological quantum dot formed using dual TICs in HgTe QWs with inverted band structures. The conductance of the system can be tuned by changing the Fermi energy, the width of the TICs (via tuning the split-gate voltages), and the QW bandgap $M$ (via tuning the bottom gate voltages and/or the thickness of the HgTe QW). Upon changing the incident Fermi energies, the transmission exhibits a series of Fano resonances originating from the Fabry-Pérot modes localized in the quantum dot, or a rather flat plateau arising from the TI edge state. In particular, with appropriate control of the Fermi energy and the TIC configurations, it is possible to trap charge carriers into the spin-polarized helical TI edge states in the quantum dot, which may pave a new path of constructing charge/spin trapping memory elements for both electronic and spintronic devices. Finally we show that the proposed topological quantum dot memory element is immune to small RSOI or magnetic fields, as well as local perturbations.

\section{Acknowledgements}

This work was supported by the MOST (Grants No. 2015CB921503, 2016YFE0110000 and 2016YFA0202300), the Opening Project of MEDIT, CAS and the Preferred Foundation of Director of IMECAS.

\section{References}

1 C. L. Kane and E. J. Mele, Phys. Rev. Lett., 2005, 95, 146802.

2 L. Fu, C. L. Kane and E. J. Mele, Phys. Rev. Lett., 2007, 98, 106803.

3 B. A. Bernevig and S. C. Zhang, Phys. Rev. Lett., 2006, 96, 106802.

4 B. A. Bernevig, T. Hughes and S. C. Zhang, Science, 2006, 314, 1757-1761.
5 M. König, S. Wiedmann, C. Brüne, A. Roth, H. Buhmann, L. W. Molenkamp, X. L. Qi and S. C. Zhang, Science, 2007, 318, 766-770.

6 W. Yang, K. Chang and S. C. Zhang, Phys. Rev. Lett., 2008, 100, 056602.

7 D. Hsieh, D. Qian, L. Wray, Y. Xia, Y. S. Hor, R. J. Cava and M. Z. Hasan, Nature, 2008, 452, 970-974.

8 Y. Xia, D. Qian, D. Hsieh, L. Wray, A. Pal, H. Lin, A. Bansil, D. Grauer, Y. S. Hor, R. J. Cava and M. Z. Hasan, Nat. Phys., 2009, 5, 398-402.

9 Y. L. Chen, J. G. Analytis, J. H. Chu, Z. K. Liu, S.-K. Mo, X. L. Qi, H. J. Zhang, D. H. Lu, X. Dai, Z. Fang, S. C. Zhang, I. R. Fisher, Z. Hussain and Z. X. Shen, Science, 2009, 325, 178-181.

10 M. Miao, Q. Yan, C. Van de Walle, W. Lou, L. Li and K. Chang, Phys. Rev. Lett., 2012, 109, 186803.

11 D. Zhang, W. Lou, M. Miao, S. Zhang and K. Chang, Phys. Rev. Lett., 2013, 111, 156402.

12 M. Z. Hasan and C. L. Kane, Rev. Mod. Phys., 2010, 82, 3045. 13 X. L. Qi and S. C. Zhang, Rev. Mod. Phys., 2011, 83, 1057.

14 D. Hsieh, Y. Xia, D. Qian, L. Wray, J. H. Dil, F. Meier, J. Osterwalder, L. Patthey, J. G. Checkelsky, N. P. Ong, A. V. Fedorov, H. Lin, A. Bansil, D. Grauer, Y. S. Hor, R. J. Cava and M. Z. Hasan, Nature, 2009, 460, 1101.

15 Z. Wu and J. Li, Nanoscale Res. Lett., 2012, 7, 90.

16 L. Z. Lin, F. Cheng, L. B. Zhang, D. Zhang and W. Yang, Phys. Rev. B: Condens. Matter Mater. Phys., 2013, 87, 245311.

17 H. Lu, A. Zhao and S. Shen, Phys. Rev. Lett., 2013, 111, 146802.

18 Z. Siu, M. Jalil and S. Tan, Sci. Rep., 2014, 4, 5062.

19 Y. Jing, S. Huang, K. Zhang, J. Wu, Y. Guo, H. Peng, Z. Liu and H. Xu, Nanoscale, 2016, 8, 1879-1885.

20 S. Bauer and C. Bobisch, Nat. Commun., 2016, 7, 11381.

21 A. Rahim, A. Levin, G. Gusev, Z. Kvon, E. Olshanetsky, N. Mikhailov and S. Dvoretsky, 2D Mater., 2015, 2, 044015.

22 C. Liu, T. Hughes, X. Qi, K. Wang and S. Zhang, Phys. Rev. Lett., 2008, 100, 236601.

23 L. Du, I. Knez, G. Sullivan and R. Du, Phys. Rev. Lett., 2015, 114, 096802.

24 S. Küfner and F. Bechstedt, Phys. Rev. B: Condens. Matter Mater. Phys., 2014, 89, 195312.

25 Q. Liu, X. Zhang, L. Abdalla and A. Zunger, Adv. Funct. Mater., 2016, 26, 3259-3267.

26 G. Zhang, X. Li, G. Wu, J. Wang, D. Culcer, E. Kaxirasf and Z. Zhang, Nanoscale, 2014, 6, 3259-3267.

27 L. Kou, B. Yan, F. Hu, S. Wu, T. Wehling, C. Felser, C. Chen and T. Frauenheim, Nano Lett., 2013, 13, 6251-6255.

28 H. Zhang, Y. Ma and Z. Chen, Nanoscale, 2015, 7, 1915219159.

29 F. Ma, G. Gao, Y. Jiao, Y. Gu, A. Bilic, H. Zhang, Z. Chen and A. Du, Nanoscale, 2016, 8, 4969-4975.

30 M. Yuan, W. Ji, M. Ren, Y. Wang and H. Zhao, RSC Adv., 2016, 6, 86089-86094.

31 X. Li, S. Zhang and Q. Wang, Nanoscale, 2017, 9, 562-569.

32 Q. Liu, X. Zhang, L. Abdalla, A. Fazzio and A. Zunger, Nano Lett., 2015, 15, 1222-1228. 
33 J. Zhou, Q. Sun, Q. Wang, Y. Kawazoed and P. Jena, Nanoscale, 2016, 8, 11202-11209.

34 S. Küfner, J. Furthmüller, L. Matthes, M. Fitzner and F. Bechstedt, Phys. Rev. B: Condens. Matter Mater. Phys., 2013, 87, 235307.

35 Y. Ma, Y. Dai, C. Niu and B. Huang, J. Mater. Chem., 2012, 22, 12587-12591.

36 A. Wang, X. Zhang and M. Zhao, Nanoscale, 2014, 6, 1115711162.

37 H. Zhao, C. Zhang, W. Ji, R. Zhang, S. Li, S. Yan, B. Zhang, P. Li and P. Wang, Sci. Rep., 2016, 6, 20152.

38 H. Zhao, W. Ji, C. Zhang, P. Li, S. Zhang, F. Li, P. Wang, S. Li and S. Yan, J. Mater. Chem. C, 2017, 5, 2656-2661.

39 D. N. Sheng, Z. Y. Weng, L. Sheng and F. D. M. Haldane, Phys. Rev. Lett., 2006, 97, 036808.

40 C. Xu and J. E. Moore, Phys. Rev. B: Condens. Matter Mater. Phys., 2006, 73, 045322.

41 C. J. Wu, B. A. Bernevig and S. C. Zhang, Phys. Rev. Lett., 2006, 96, 106401.

42 L. Fu and C. L. Kane, Phys. Rev. B: Condens. Matter Mater. Phys., 2007, 76, 045302.

43 B. J. van Wees, H. vanHouten, C. W. J. Beenakker, J. G. Williamson, L. P. Kouwenhoven, D. van der Marel and C. T. Foxon, Phys. Rev. Lett., 1988, 60, 848.

44 D. A. Wharam, T. J. Thornton, R. Newbury, M. Pepper, H. Ahmed, J. E. F. Frost, D. G. Hasko, D. C. Peacock, D. A. Ritchie and G. A. C. Jones, J. Phys. C: Solid State Phys., 1988, 21, L209.
45 D. Loss and D. P. DiVincenzo, Phys. Rev. A, 1988, 57, 120.

46 A. Imamoglu, D. D. Awschalom, G. Burkard, D. P. DiVincenzo, D. Loss, M. Sherwin and A. Small, Phys. Rev. Lett., 1999, 83, 4204.

47 R. Hanson, L. P. Kouwenhoven, J. R. Petta, S. Tarucha and L. M. K. Vandersypen, Rev. Mod. Phys., 2007, 79, 1217.

48 M. A. Kastner, Rev. Mod. Phys., 1992, 64, 849.

49 A. Galindo and M. A. M. Delgado, Rev. Mod. Phys., 2002, 74, 347.

50 B. Zhou, H. Lu, R. Chu, S. Shen and Q. Niu, Phys. Rev. Lett., 2008, 101, 246807.

51 J. C. Y. Teo and C. L. Kane, Phys. Rev. B: Condens. Matter Mater. Phys., 2009, 79, 235321.

52 A. Ström and H. Johannesson, Phys. Rev. Lett., 2009, 102, 096806.

53 V. Krueckl and K. Richter, Phys. Rev. Lett., 2011, 107, 086803. 54 C. W. Huang, S. T. Carr, D. Gutman, E. Shimshoni and A. D. Mirlin, Phys. Rev. B: Condens. Matter Mater. Phys., 2013, 88, 125134.

55 L. B. Zhang, F. Cheng, F. Zhai and K. Chang, Phys. Rev. B: Condens. Matter Mater. Phys., 2011, 83, 081402(R).

56 J. Klinovaja and D. Loss, Phys. Rev. B: Condens. Matter Mater. Phys., 2015, 92, 121410(R).

57 J. P. Bird, Electron Transport in Quantum Dots, Springer Science+Business Media, New York, 2003.

58 Z. H. Wu, F. M. Peeters and K. Chang, Phys. Rev. B: Condens. Matter Mater. Phys., 2010, 82, 115211.

59 K. Chang and W. K. Lou, Phys. Rev. Lett., 2016, 106, 206802. 